\title{
Validation of Methods for the Optical Characterisation of the Wettability of Polymeric Films for Food Packaging
}

\author{
Giulio D'Emilia and Emanuela Natale \\ Dipartimento di Ingegneria Industriale e dell'Informazione e di Economia (DIIIE), University of L'Aquila, Via G. Gronchi 18, \\ Loc. Campo di Pile, 67100 L'Aquila, Italy \\ Correspondence should be addressed to Emanuela Natale; emanuela.natale@univaq.it
}

Received 9 May 2014; Revised 10 October 2014; Accepted 13 October 2014; Published 23 October 2014

Academic Editor: Gabor Szederkenyi

Copyright ( 2014 G. D’Emilia and E. Natale. This is an open access article distributed under the Creative Commons Attribution License, which permits unrestricted use, distribution, and reproduction in any medium, provided the original work is properly cited.

\begin{abstract}
A methodology is described for the theoretical-experimental evaluation of the measurement uncertainty of the polar and dispersive components of the surface free energy (SFE) in polypropylene films; these parameters are related to the film wettability of adhesives and inks. The proposed method is based on the measurement by means of a vision system of the contact angles of liquid drops deposited on the film itself, which allows for obtaining, through mathematical models drawn from the literature, the physical quantities of interest. The effect of the principal influence parameters has been experimentally evaluated, and testing has allowed the defining of the technical procedures readily transferable in the industry. The uncertainty assessment is interesting not only to correctly evaluate experimental data but also to characterise the reproducibility of the effects of techniques for improving the wettability of films, such as surface treatments.
\end{abstract}

\section{Introduction}

The possibility of measuring the wettability of polymeric films, particularly of polypropylene materials for food packaging applications, is becoming increasingly important from an industrial and economic standpoint because it determines the possibility of using new materials for bonding and new inks for printing. These technological innovations, provided they ensure a satisfactory and stable surface adhesion, allow for creating packaging that is increasingly pleasing from an aesthetic point of view and for geometries of packaging that are more and more particular, as the food market requires. If, instead, the characteristics of wettability of adhesives are taken into consideration, their better characterisation allows for reducing the area of overlap for the gluing and consequently to reduce the film surface required by more than $10 \%$, as experimentally verified. The wettability is the technological characteristic that takes into account the capacity of adhesion of a liquid on a solid film; the physical quantities to evaluate it are the polar and dispersive components of the surface free energy (SFE), usually expressed in millinewtons per meter, for which there are no methods for direct measurement.
The polar SFE, constituting together with the dispersive component the overall SFE, has greater effect on the wettability of polypropylene films; the methods currently in use, as defined in ASTM D 2578 [1], that provide the overall SFE are, thus, only partially exhaustive. They, in fact, are not always able to provide the information necessary to support the experimental investigations, which are usually performed to characterise the film produced and to assess the effect of surface treatments to increase the wettability. The results that can be obtained with the procedure proposed in [1] are also significantly affected by the subjectivity of the operator assessment, and the method provides the SFE only as a value belonging to predefined classes.

However, different methods are already available in the literature for the calculation of the SFE and the significant components of the phenomenon. In fact, there are the methods of Fox and Zisman [2] and the method of Fowkes [3] capable of providing the polar and disperse components in two subsequent steps of the calculation, which are then reduced to one from Owens-Wendt-Rabel and Kaeble (OWRK), as described in [4]. 
They provide different physical models that bind the quantities of interest, even if their validity appears limited to certain classes of materials (polymeric films with different treatments, metals, paints, coatings, and biological film), due to the specific modelling assumptions. All, however, make it possible to evaluate the polar and disperse components of the SFE, by direct measurement of the contact angle; consequently, the uncertainties with which the polar and disperse SFE can be assessed depend on the measurement uncertainty of the contact angle.

In this paper, particular reference is made to the method (OWRK) [4] among the methods available in the literature for several reasons: the physical hypotheses appear complete and suitable to the type of polymer studied, and the approach is operationally feasible, even in an industrial context. For these reasons, it has been used in other works described in the literature [5-7].

Although the method is widespread, preliminary evaluation of the measurement uncertainty only recently appeared $[4,5,7]$. In particular, reference [7] proposes an analytical evaluation of the SFE uncertainty. In this paper, however, an experimental uncertainty assessment is proposed, which takes into account the real behavior of the instrument used.

The measurement uncertainty of the methodology and also the identification of technical-operational solutions to reduce it appear important, not only for reasons of metrological correctness but also to readily identify possibilities of the development of new materials and the effects of surface treatments.

In fact, as will be described below, the method is affected by numerous influence variables of different types, which complicates the uncertainty evaluation.

\section{The Physical Model and the Experimental Methodology}

The general equation describing the interaction of the surface tension of a liquid and a solid is called the Young equation [4]:

$$
\gamma_{s}=\gamma_{\mathrm{sl}}+\gamma_{\mathrm{lv}} \cos \theta
$$

where $\theta$ is the measured contact angle, $\gamma_{s}$ is the surface free energy (SFE) of the solid in the case of negligible spreading pressure, and $\gamma_{\mathrm{sl}}$ and $\gamma_{\mathrm{lv}}$ are the surface tensions at the solidliquid and liquid-vapour interface, respectively. Both the surface tension and the SFE can be split into two components considering only the dispersion forces on the one hand and all polar interactions on the other:

$$
\gamma=\gamma^{d}+\gamma^{p}
$$

On the basis of this approach, Owens, Wendt, Rabel, and Kaeble developed a linear equation [4], in which the slope and the intercept are given by the square root of both the polar and the disperse components of the SFE, respectively:

$$
0.5 \cdot \gamma_{\mathrm{lv}} \cdot \frac{(\cos \theta+1)}{\left({\gamma_{\mathrm{lv}}}^{d}\right)^{0.5}}=\left({\gamma_{s}}^{p}\right)^{0.5} \cdot\left(\frac{{\gamma_{\mathrm{lv}}}^{p}}{{\gamma_{\mathrm{lv}}}^{d}}\right)^{0.5}+\left({\gamma_{s}}^{d}\right)^{0.5}
$$

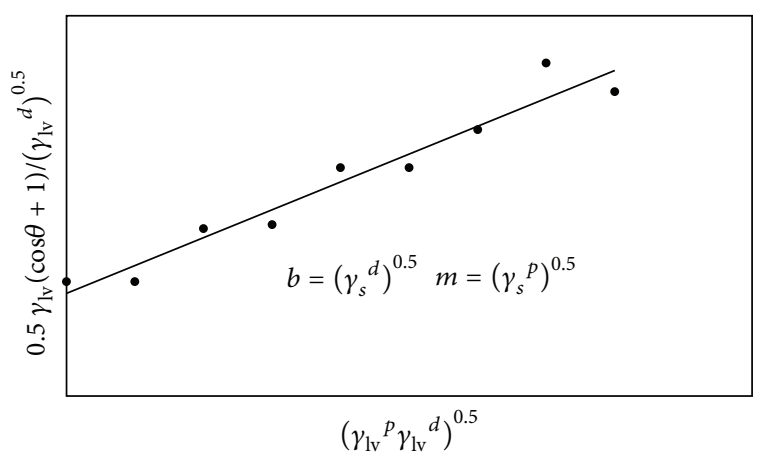

FIGURE 1: Method for the determination of the polar and disperse parts of the surface free energy, where $m$ and $b$ are the slope and intercept, respectively, of the best fit line of the experimental data [4].

Thus, the measurement of the contact angles of different liquids, with known surface tensions and relative components, enables the determination of the polar and disperse parts of SFE by the line of best fit (least square method) of the experimental data (Figure 1).

The knowledge of the values of the polar and disperse components of the SFE allows for describing a curve, very important from the point of view of applications, the "wetting envelope" [4], which makes particularly immediate the evaluation of the surface wettability by a known liquid. If the representative point of the new liquid is inside the curve, the film will be "wetted."

\section{Materials, Measurement Method, and Results}

As previously mentioned, for the indirect evaluation of the SFE components of a polypropylene film, the method of the contact angle measurement has been used, using a vision system, which allows the assessment of the geometrical parameters of the "sessile" drop, both statically and dynamically, where the dynamic measure consists of the determination of the temporal trend of different geometric parameters of interest, such as the contact angle and volume of the drop and position of the contact line.

The measurements have been conducted on a test bench consisting of a commercial system for the analysis of images, OCA20 of Data Physics Company, which has been used to dispense, in a controlled manner, the volumes of the drops and to monitor their variation over time, to optimise the fixing of the film and to adjust the illumination of the sample. A part of the image processing has been performed by the software package of the above measurement system; some procedures for image and data processing have also been specifically developed in this work to achieve both a validation of the software indications and different information for comparison purposes.

Two different polypropylene films have been analysed, Film1 and Film2, for reasons of industrial privacy. They are 
both PET films, but the second has been subjected to a corona surface treatment [6].

The analysis of the drop geometry has been conducted by comparing the results of three different geometric models:

(a) circular fitting of the drop outline (sphericity assumption);

(b) Laplace curve fitting;

(c) $\theta / 2$ method, taking into account the appropriate corrections [8].

The sample fluids were water, formamide, and diiodomethane.

For the surface tensions, the following values have been assumed $[4,7]$ :

water: $\gamma_{\mathrm{lv}}=72.8 \mathrm{mN} / \mathrm{m} ;{\gamma_{\mathrm{lv}}}^{d}=21.8 \mathrm{mN} / \mathrm{m} ; \gamma_{\mathrm{lv}}{ }^{p}=$ $51.0 \mathrm{mN} / \mathrm{m}$;

formamide: $\gamma_{\mathrm{lv}}=58.0 \mathrm{mN} / \mathrm{m} ;{\gamma_{\mathrm{lv}}}^{d}=39.0 \mathrm{mN} / \mathrm{m} ; \gamma_{\mathrm{lv}}{ }^{p}$ $=19.0 \mathrm{mN} / \mathrm{m}$;

diiodomethane: $\gamma_{\mathrm{lv}}=50.8 \mathrm{mN} / \mathrm{m} ; \gamma_{\mathrm{lv}}{ }^{d}=49.5 \mathrm{mN} / \mathrm{m}$; $\gamma_{\mathrm{lv}}{ }^{p}=1.3 \mathrm{mN} / \mathrm{m}$.

The percentage uncertainties of these data have been taken equal to $2 \%$ of the total component, according to the literature indications [4].

The sample fluids have been chosen because they wet the polypropylene film in a unique way; the wettability decreases rapidly from diiodomethane to formamide and water. The contact angle follows an opposite trend.

The main sources of uncertainty in the contact angle measurement can be classified into the following types [4]:

(1) operational, such as drop volume, waiting time between the drop deposition and the angle measurement, temperature, and humidity;

(2) instrumental, as the variables related to the image optical analysis for the determination of the geometric parameters of the drop, such as angle and volume;

(3) accuracy of the surface tension values of liquids used;

(4) accuracy of the used model;

(5) physical inhomogeneity (roughness and stretching due to the production process and the superficial treatment) and chemical inhomogeneity of the film surface.

In this work, the uncertainty will be evaluated in terms of the standard uncertainty, $s$.

Tests have been conducted with the aim of identifying the different contributions that will be afterwards composed.

3.1. Operational and Instrumental Factors. Graphs of Figures 2,3 , and 4 show some of the results of tests conducted by varying some parameters of interest, in particular, the size of the drop and the waiting time between the drop deposition and the angle measurement for different films and reference liquids. The drop outline has been obtained by using the circular fitting method.

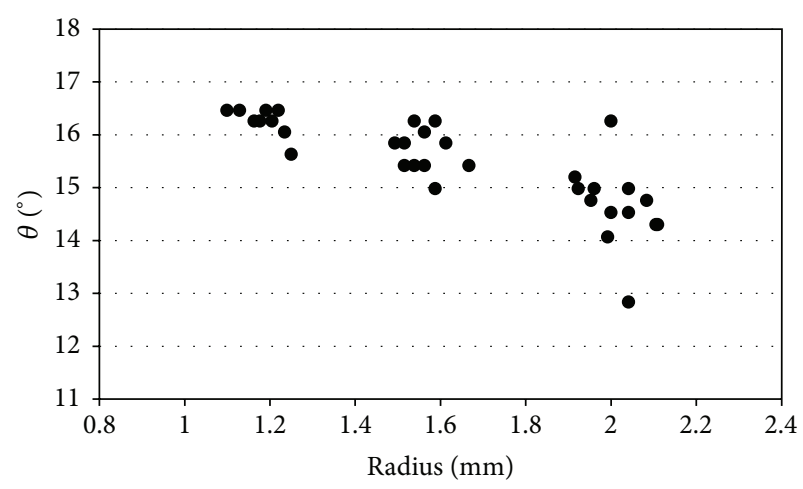

Figure 2: Dependence of $\theta$ on the radius of the drop for the pair Filml-diiodomethane, assuming a circular model for the drop outline.

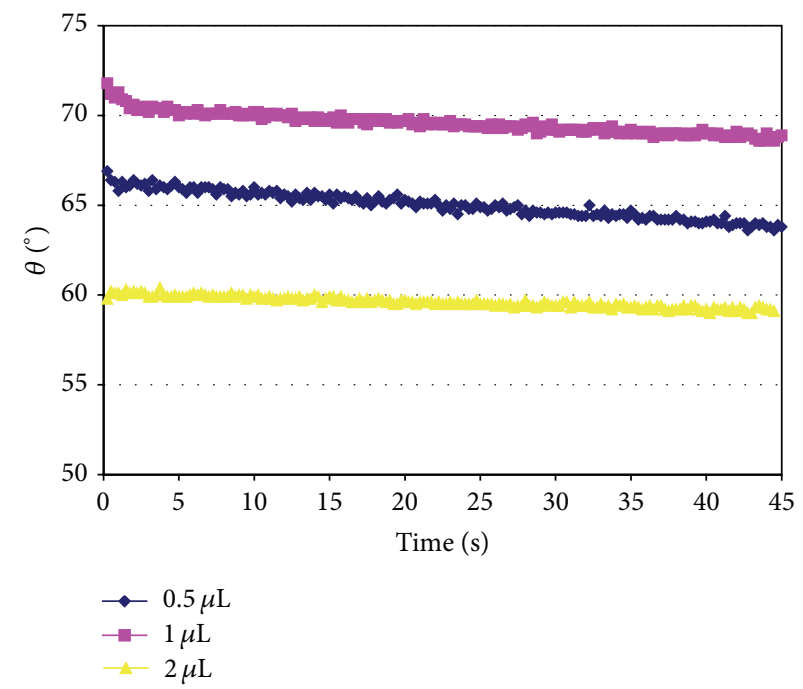

Figure 3: Variation over time of the contact angle, for the pair Film2-water (drop volumes: $0.5,1,2.0 \mu \mathrm{L}$ ).

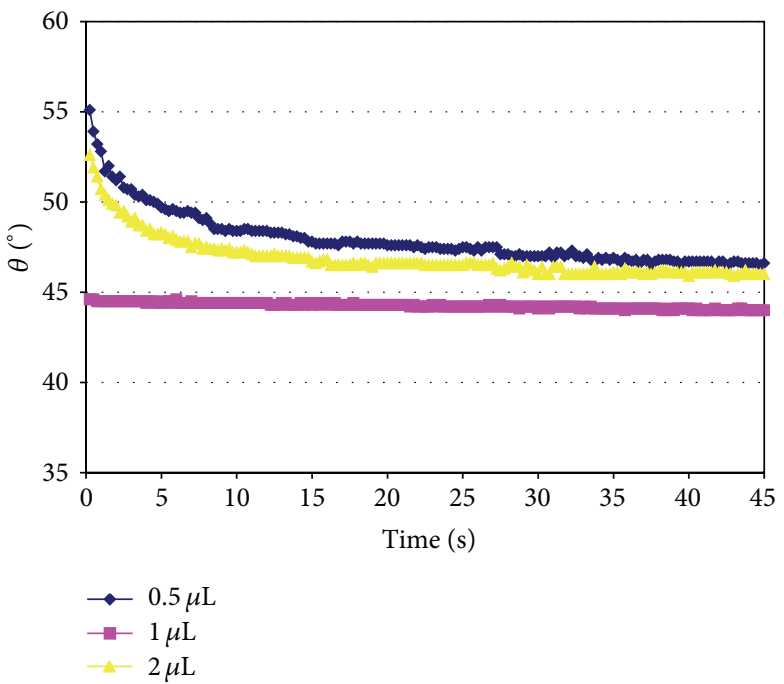

FIgURE 4: Variation over time of the contact angle, for the pair Film2-diiodomethane (drop volumes: $0.5,1,2.0 \mu \mathrm{L}$ ). 


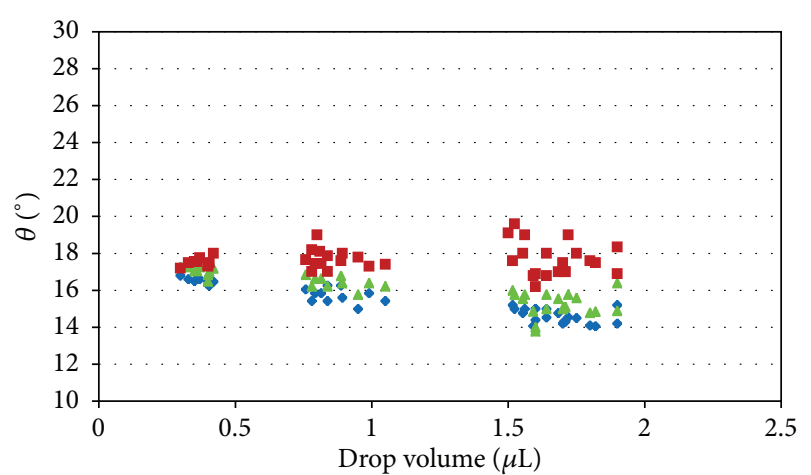

- Circular model

$\Delta \theta / 2$ model

- Laplace model

Figure 5: Dependence of the contact angle on the drop volume and on the method used to define the drop outline, for the pair Film1diiodomethane.

The diagrams refer to different pairs of film-liquid, not for the purpose of comparison, but with the aim to give examples of the data from which the contribution of the different variables involved has been calculated.

The effect of size (Figure 2) appears more important on the measurement uncertainty of the angle, with respect to the waiting time (Figures 3 and 4). In fact, as long as you wait a minimum time for the settling of the drop, which is dependent on the liquid, the percentage changes of data over time appear to be less important than the other effects.

The tests have been conducted in laboratory with the temperature in the range $(20 \pm 2)^{\circ} \mathrm{C}$ and relative humidity in the field $(50 \pm 10) \%$. The effects of these parameters, which affect the liquid evaporation, have been taken into account experimentally by evaluating the temporal trend of the contact angle. They appear, however, to be not relevant.

The graphs of Figures 3 and 4 show that the measurement dispersion also depends on the type of film/fluid pair; the highest variability occurs with the water, to which the films of polypropylene must be strongly repellent.

Figure 5 shows the variation of the results when different methods of fitting are used, according to different geometric hypotheses.

Fifty tests have been conducted for each film/liquid pair with different operators, on different days, in the environmental conditions specified above, for data reproducibility evaluation.

The contribution to the overall uncertainty of the environmental and operating variables, such as, in particular, volume and modelling of the drop outline, has been expressed as standard deviation $s_{\mathrm{vm}}$ of the results.

The contribution to the overall uncertainty of the waiting time before the angle measure has been evaluated as the standard deviation $s_{t}$, assuming a rectangular probability distribution between the minimum and the maximum value of a measurement time sequence.

The use for testing of different samples of the same type of film has allowed for taking into account the variability of

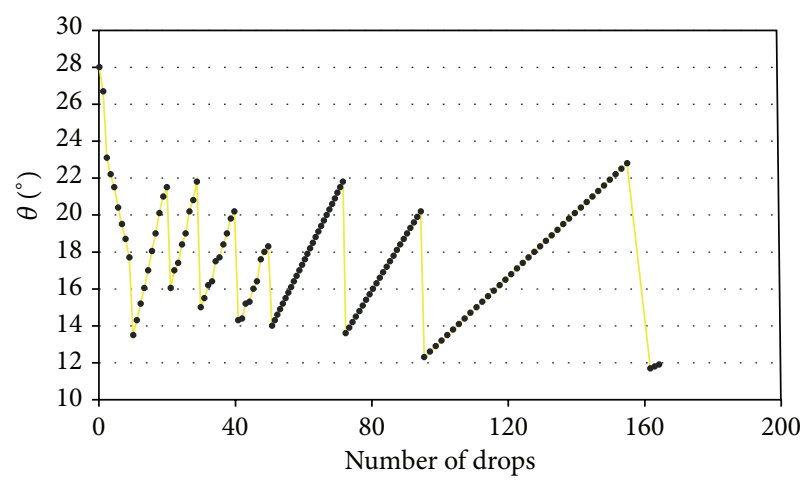

FIGURE 6: Temporal trend of the contact angle, for the pair Film1diiodomethane, when the drop volume increases with continuity; in abscissa, the progressive number of drops dispensed. Maximum volume $=20 \mu \mathrm{L}$.

the surface roughness and inhomogeneity related to surface treatments.

3.2. Other Factors. Considering the period of validity of the certificated properties of liquids and the test duration, the effect of the stability of the reference fluids has been considered negligible.

3.3. Uncertainty Assessment. The overall uncertainty has been obtained according to [9] by the following equation:

$$
s^{2}=s_{\mathrm{vm}}^{2}+s_{t}^{2} .
$$

The uncertainty changes, as outlined above, depending on the film/fluid pair considered.

For example, in the case of Film1/diiodomethane $\theta=$ $(16.1 \pm 1.2)^{\circ}$, and in the case of Film $2 /$ water $\theta=(69.2 \pm 4.0)^{\circ}$. The other values of $\theta$ are intermediate.

A confirmation of the variability estimated for the contact angles has been obtained from dynamic tests; the temporal trend of $\theta$ is evaluated when the volume is increased continuously, beyond the value of the maximum value of the drop volume $(2 \mu \mathrm{L})$ set in the static tests. The results of this type of test are affected by hysteresis phenomena [10], as shown by the "sawtooth" trend of the diagram in Figure 6.

In this situation, metastable conditions of the drop occur, and the variability of $\theta$ appears overestimated.

It is noted that, considering an average hysteresis interval of $6^{\circ}$ for the Filml, as can be seen from the graph in Figure 6, and assuming a rectangular probability distribution within this range, the resulting uncertainty $u_{\text {hyst }}$ can be evaluated as [9]

$$
u_{\text {hyst }}=\frac{6^{\circ}}{2 \sqrt{3}}=1.7^{\circ} .
$$

This value of uncertainty seems to be overestimated, as mentioned, and, in fact, it is assumed to be a confirmation of the uncertainty evaluation of $1.2^{\circ}$, as evaluated for the Filml.

The measure of the $\theta$ values and the evaluation of their uncertainties have allowed for assessing, in accordance with 


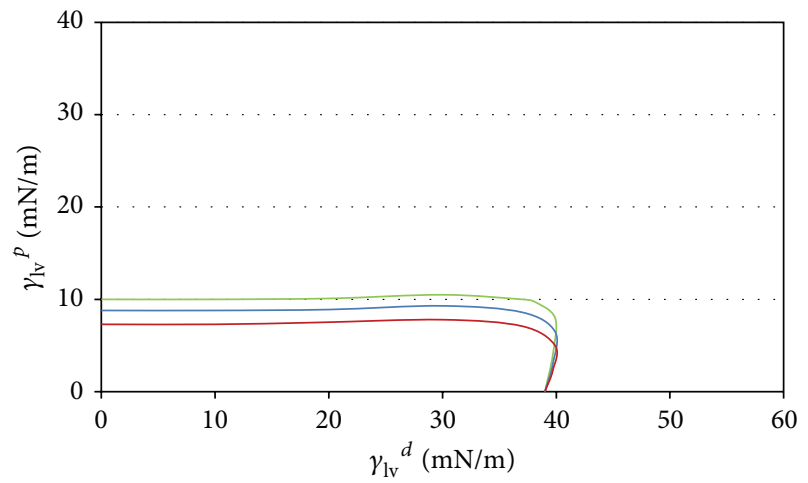

FIGURE 7: Wetting envelope for Film1. In abscissa, the disperse component, $\gamma_{\mathrm{lv}}{ }^{d}$, and in ordinate the polar component, $\gamma_{\mathrm{lv}}{ }^{p}$, of surface tension. The uncertainty range is represented $\left(u\left(\gamma_{\mathrm{lv}}{ }^{p}\right)=\right.$ $1.5 \mathrm{mN} / \mathrm{m}, u\left(\gamma_{\mathrm{lv}}{ }^{d}\right)$ negligible).

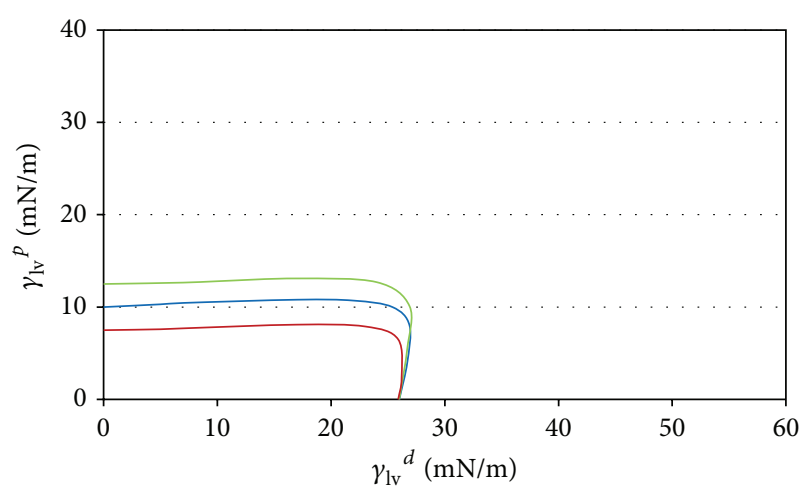

FIGURE 8: Wetting envelope for Film2. In abscissa, the disperse component, $\gamma_{\mathrm{lv}}{ }^{d}$, and in ordinate, the polar component, $\gamma_{\mathrm{lv}}{ }^{p}$, of surface tension. The uncertainty range is represented $\left(u\left(\gamma_{\mathrm{lv}}{ }^{p}\right)=\right.$ $2.5 \mathrm{mN} / \mathrm{m}, u\left({\gamma_{\mathrm{lv}}}^{d}\right)$ negligible).

(3), the components $\gamma_{s}^{p}$ and $\gamma_{s}^{d}$ for the two films studied, when subjected to different surface treatments.

The uncertainties of SFE have been obtained by evaluating the values of $\gamma_{s}{ }^{p}$ and $\gamma_{s}{ }^{d}$ by the model described, varying $\theta$ in the range of uncertainty $(\theta \pm s)^{\circ}$, and calculating the standard deviation of the data. The effect of the uncertainty of the characteristics of the reference fluids appears to be negligible.

If the results are taken into account, the component affected by greater uncertainty is $\gamma_{s}^{p}$.

In particular, $\gamma_{s}^{p}=(7.0 \pm 1.3) \mathrm{mN} / \mathrm{m}$ in the case of Film1 and $\gamma_{s}{ }^{p}=(10.4 \pm 2.1) \mathrm{mN} / \mathrm{m}$ in the case of Film2.

In Figures 7 and 8 , the curves of wettability in the wettability diagram are described, obtained for Film1 and Film2 and calculated in correspondence with the aforementioned average angles $\theta=16.1$ and $\theta=69.2$, respectively; the uncertainty range is also represented $\left(u\left(\gamma_{\mathrm{lv}}{ }^{p}\right)=1.5 \mathrm{mN} / \mathrm{m}\right.$ and $u\left(\gamma_{\mathrm{lv}}{ }^{d}\right)$ negligible in the case of Filml, $u\left(\gamma_{\mathrm{lv}}{ }^{p}\right)=2.5 \mathrm{mN} / \mathrm{m}$ and $u\left(\gamma_{\mathrm{lv}}{ }^{d}\right)$ negligible in the case of Film2).

There is a need to "stress" the surface treatment for the Film2 to create a sufficient area of wettability in the wettability diagram. The low value of dispersive SFE, $\gamma_{s}{ }^{d}$, has led to a considerable increase of the uncertainty, presumably due to higher physical and chemical nonuniformity of the surface, due to the stressing surface treatment.

These results suggest that the average value of the SFE provided by the measurement method is not sufficient to make assessments about the wettability of films, especially when they are subjected to surface treatments to increase the wettability itself. Uncertainty evaluation is, then, essential to make reliable evaluations of the possible compatibility of new materials.

\section{Conclusions}

In the present work, the effect of the influence variables in the measurement of the contact angle of drops, $\theta$, deposited on different polypropylene films for food packaging was evaluated.

In particular, the effect of the operational quantities was evaluated, such as drop size, growth rate, waiting time, positioning of the sample on the measuring bench, lighting, and geometric modelling of the droplet.

The analysis enables the identification of the most important influence parameters, which is also useful for the implementation of experimental procedures that are transferable in an industrial contest.

The study of different films allowed for highlighting the importance of the surface treatment on the measurement accuracy. This treatment, on one hand, leads to improvement of the wettability. On the other hand, because of the possible considerable increase of the measurement uncertainty of SFE, it may affect the possibility to provide operatively usable information based on experimental data having an excessive measurement uncertainty.

\section{Conflict of Interests}

The authors declare that there is no conflict of interests regarding the publication of this paper.

\section{References}

[1] "Standard test method for wetting tension of polyethylene and polypropylene films," Tech. Rep. ASTM D2578-09, ASTM, 2009.

[2] H. W. Fox and W. A. Zisman, "The spreading of liquids on lowenergy surfaces. II. Modified tetrafluoroethylene polymers," Journal of Colloid Science, vol. 7, no. 2, pp. 109-121, 1952.

[3] F. M. Fowkes, "Role of acid-base interfacial bonding in adhesion," Journal of Adhesion Science and Technology, vol. 1, no. 1, pp. 7-27, 1987.

[4] G. Cappelletti, S. Ardizzone, D. Meroni et al., "Wettability of bare and fluorinated silanes: a combined approach based on surface free energy evaluations and dipole moment calculations," Journal of Colloid and Interface Science, vol. 389, no. 1, pp. 284291, 2013.

[5] R. B. Shaevich, "Measurement of the specific free surface energy of solids," Measurement Techniques, vol. 50, no. 10, pp. 1121-1123, 2007. 
[6] I. Vlaeva, T. Yovcheva, A. Viraneva et al., "Contact angle analysis of corona treated polypropylene films," Journal of Physics: Conference Series, vol. 398, no. 1, Article ID 012054, 2012.

[7] A. Rudawska and E. Jacniacka, "Analysis for determining surface free energy uncertainty by the Owen-Wendt method," International Journal of Adhesion and Adhesives, vol. 29, no. 4, pp. 451-457, 2009.

[8] M. W. Yang and S. Y. Lin, "A method for correcting the contact angle from the $\theta / 2$ method," Colloids and Surfaces A: Physicochemical and Engineering Aspects, vol. 220, pp. 199-210, 2003.

[9] "Uncertainty of measurement-part 3: guide to the expression of uncertainty in measurement," ISO/IEC Guide 98-3(2008), 1995.

[10] H. B. Eral, D. J. C. M. 't Mannetje, and J. M. Oh, "Contact angle hysteresis: a review of fundamentals and applications," Colloid and Polymer Science, vol. 291, no. 2, pp. 247-260, 2013. 

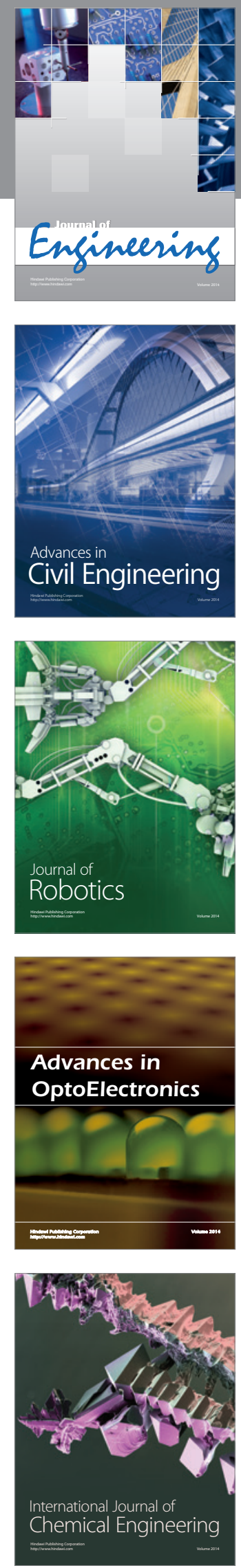

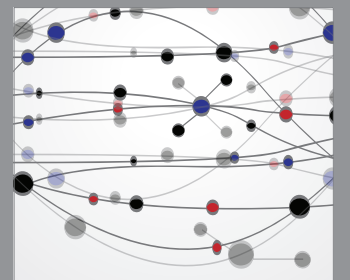

The Scientific World Journal
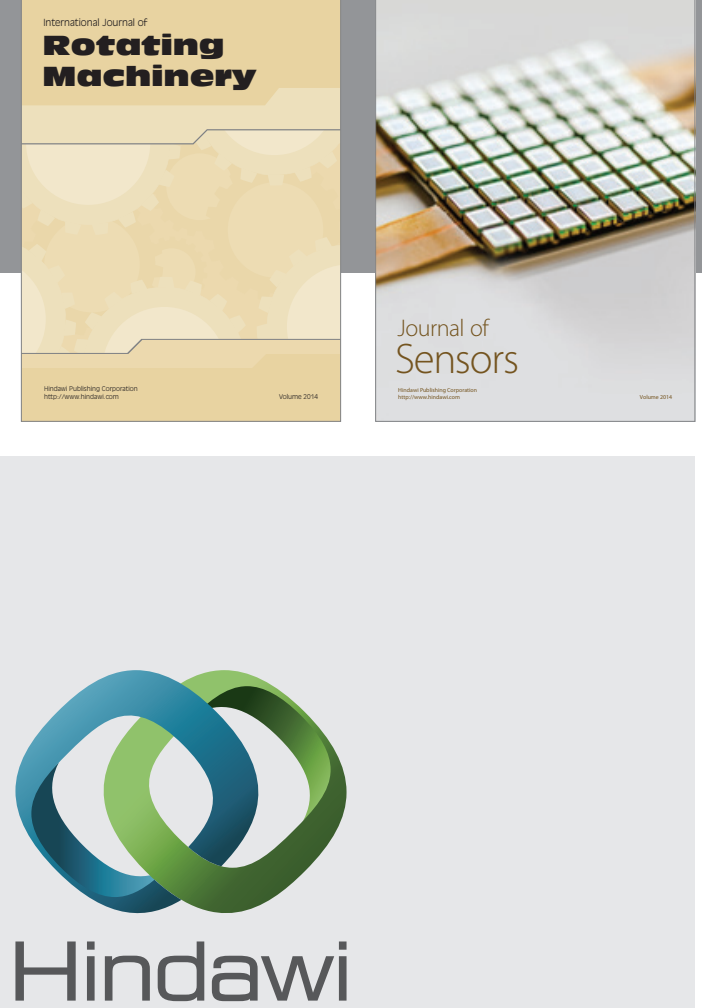

Submit your manuscripts at http://www.hindawi.com
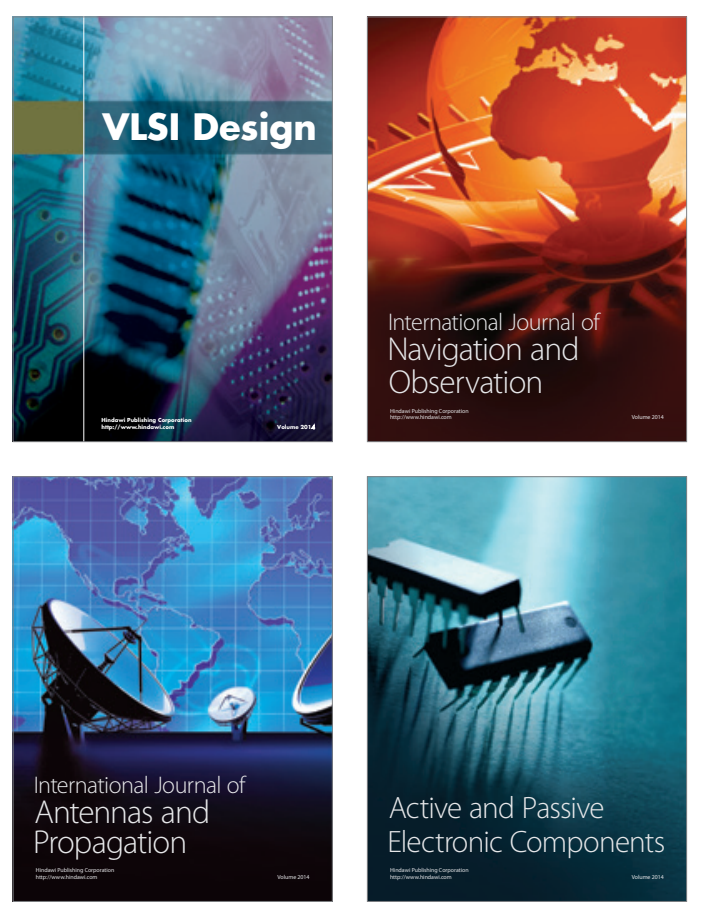
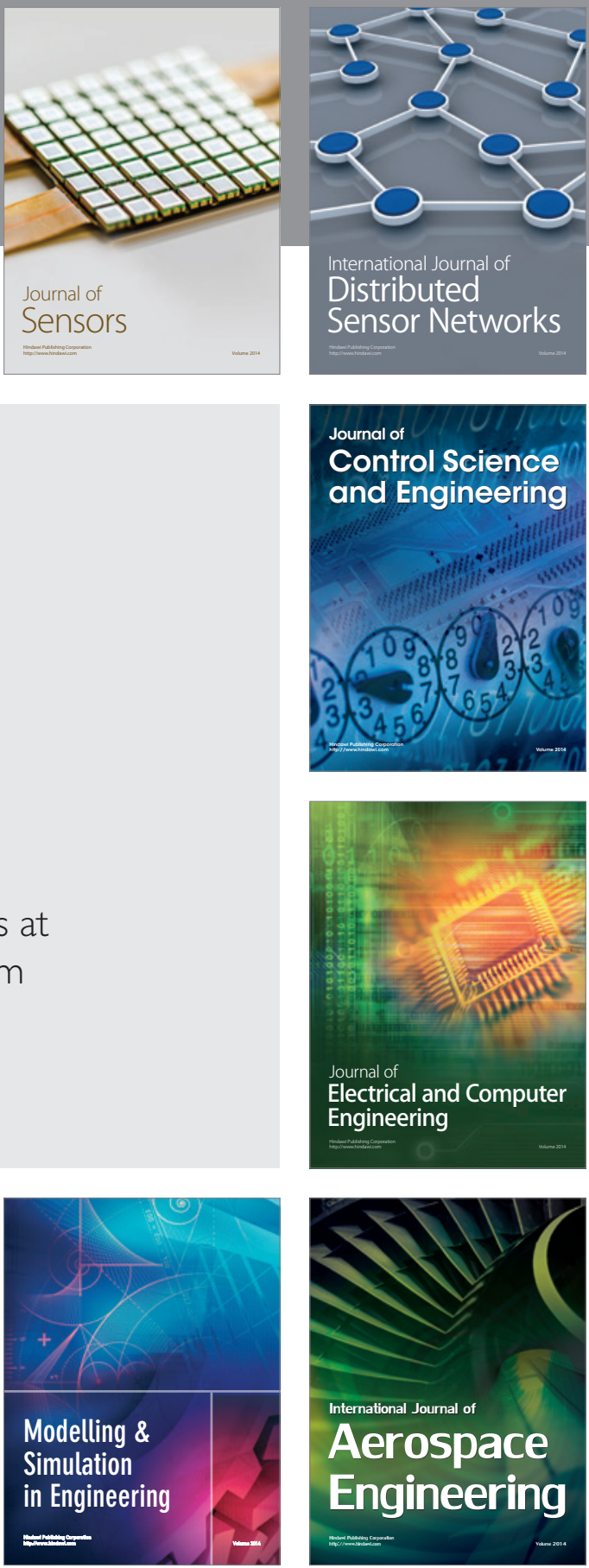

Journal of

Control Science

and Engineering
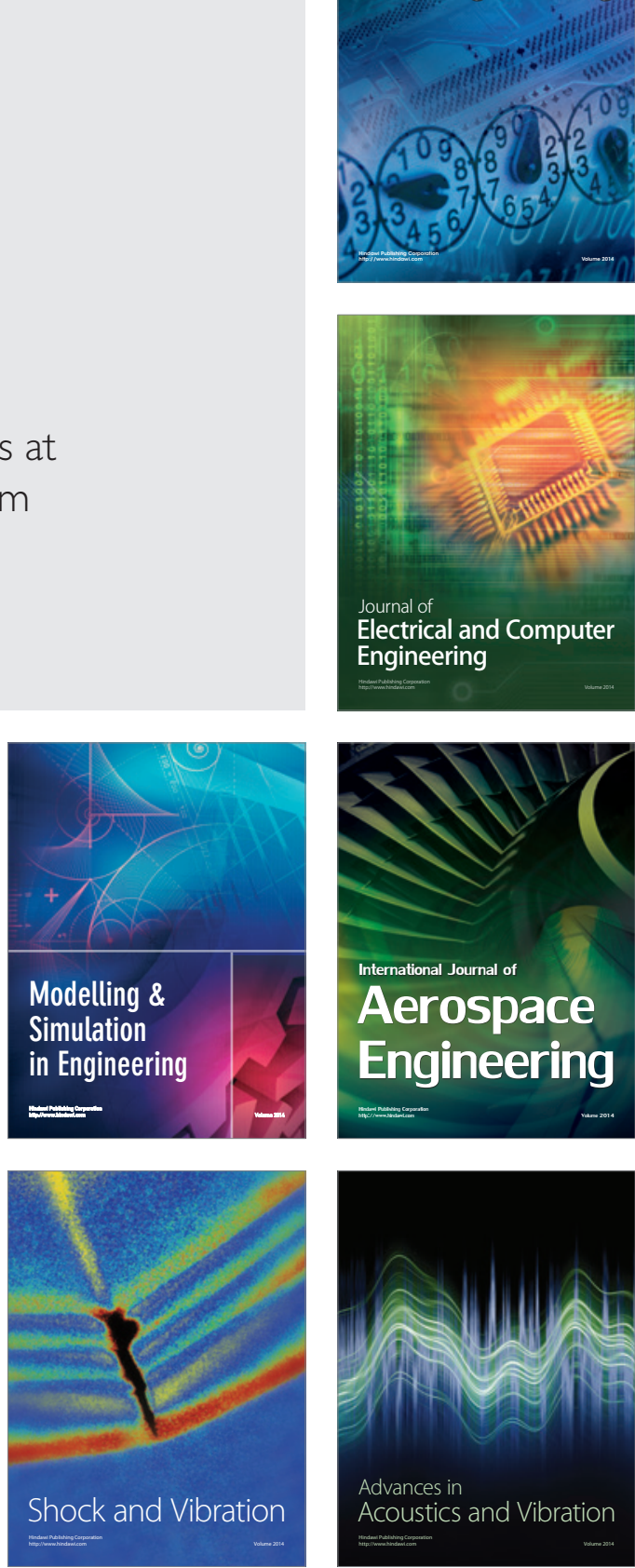